\title{
El método científico como estrategia pedagógica para activar el pensamiento crítico y reflexivo*
}

\author{
Luisa Mercedes Cuesta Moreno \\ Institución Educación Normal Superior Manuel Cañizales, Quibdó, Colombia \\ luisam093@gmail.com
}

\section{RESUMEN}

Se presentan los resultados y hallazgos de una intervención a partir del método científico como estrategia pedagógica. El objetivo es examinar su influencia para activar el pensamiento crítico y reflexivo en estudiantes de grado sexto, fortalecer competencias en la enseñanza de las ciencias naturales, relacionarla con fenómenos empíricos, y mejorar los procesos cognitivos.

Se utilizó un tipo de investigación mixta con triangulación de la información a partir de las técnicas aplicadas en la recogida de datos: encuestas, talleres, grupos de discusión y observación. Se aplicó un ciclo de aprendizaje titulado Ciencia y método científico el cual estuvo direccionado a facilitar el conocimiento y la comprensión de este método, que las estudiantes emplearon para explicar la maduración del aguacate en condiciones no climatéricas y la oxidación de las frutas. Uno de los hallazgos es que este método, como estrategia de trabajo en el aula, posibilita un aprendizaje duradero, significativo, es transversal, permite la fijación de conceptos y dinamiza la incorporación de nuevo conocimiento.

Palabras clave: método científico; pensamiento crítico y reflexivo; procesos cognitivos; habilidades investigativas.

\footnotetext{
Cómo citar: Cuesta Moreno, L. M. (2019). El método científico como estrategia pedagógica para activar el pensamiento crítico y reflexivo. Ciencias Sociales y Educación, 8(15), 87-104. DOI: https://doi.org/10.22395/ csye.v8n15a5

Recibido: 10 de diciembre de 2018.

Aprobado: 17 de mayo de 2019.
} 


\section{The Scientific Method as a Pedagogical Strategy to Activate Critical and Reasoned Thinking}

\section{ABSTRACT}

In this article are presented the results and findings of an intervention with the scientific method as a pedagogical strategy. The goal is to examine its influence on the activation of critical and reflective thinking in students of sixth grade, strengthening abilities in the teaching of natural sciences, link it to empiric phenomena and improve the cognitive processes.

A mixed type of research was used alongside a triangulation of information from techniques applied in data collecting: polls, workshops, discussion groups, and observation. A learning cycle called Science and the scientific method was applied which was focused on facilitating the knowledge and comprehension of this method, which was used by students for explaining the ripening of avocados in non-climacteric and the oxidation of fruits. One of the findings is that this method, as an in-class work strategy, allows long-lasting learning, as well as a significative, transversal and that allows the fixation of concepts while also dynamizes the embodiment of new knowledge.

Keywords: scientific method; critical and reflexive thinking; cognitive processes; research abilities.

\section{O método científico como estratégia pedagógica para ativar o pensamento crítico e refiexivo}

\section{RESUMO}

São apresentados os resultados e os achados de uma intervenção a partir do método científico como estratégia pedagógica. $\mathrm{O}$ objetivo é examinar sua influência para ativar o pensamento crítico e reflexivo em estudantes do sexto ano, fortalecer competências no ensino das ciências naturais, relacioná-lo com fenômenos empíricos e melhorar os processos cognitivos. Foi utilizado um tipo de pesquisa mista, com triangulação da informação a partir das técnicas aplicadas na coleta de dados: pesquisas, oficinas, grupos de discussão e observação. Foi aplicado um ciclo de aprendizagem intitulado Ciência e método científico, dirigido a facilitar o conhecimento e a compreensão desse método, que as estudantes empregaram para explicar o amadurecimento do abacate em condições não climáticas e a oxidação das frutas. Um dos achados desse método, como estratégia de trabalho em sala de aula, possibilita uma aprendizagem duradoura, significativa e transversal; além disso, permite a fixação de conceitos e torna dinâmica a incorporação de novo conhecimento.

Palavras-chave: método científico; pensamento crítico e reflexivo; processos cognitivos; habilidades investigativas. 


\section{Introducción}

Tradicionalmente nuestros estudiantes han sido formados con mayor afinidad al rol de receptores que de interlocutores con modelos sin proyección de durabilidad, donde las preguntas en el aula de clase se coartan o se limitan a preguntas cerradas o fácticas. De esta manera, se restringe la entrada a preguntas abiertas que dirijan a los estudiantes a explorar sus dudas, curiosidad, asombro, imaginación, deseo de saber y de aventurarse a cambiar una pregunta estéril por un torrente de preguntas que inquieten y lleven a pensar como resultado de un pensamiento crítico y reflexivo que promueva la acción, la autonomía y criterio para decidir. Donde los estudiantes reciban y acepten sugerencias, pero rechacen la manipulación.

\section{Considerando que:}

los estándares básicos de competencias en ciencias naturales buscan que el estudiante desarrolle las habilidades científicas y las actitudes requeridas para explorar hechos y fenómenos; analizar problemas; observar y obtener información; definir, utilizar y evaluar diferentes métodos de análisis, compartir los resultados, formular hipótesis y proponer las soluciones. (Mineducación, 2004, p. 1)

Cuando se busca formar estudiantes con un pensamiento crítico y reflexivo, se habla de una educación y formación orientada al desarrollo de habilidades y destrezas que les proporcionen seguridad y autonomía para tomar decisiones bajo un criterio de análisis auténtico. En otras palabras, que aprendan, relacionen, integren y apliquen los conceptos, en sus experiencias empíricas.

Este método permite que los estudiantes acudan al análisis de situaciones y hechos vivenciales, así como hacer predicciones sobre los resultados, las pruebas experimentales y las conclusiones estructuradas e integradoras del aprendizaje; "enfocando el desarrollo de capacidades reflexivas, críticas y pensamiento de alto nivel" (Díaz, 2003, p. 8). De esta manera facilita la proximidad a la resolución de problemas donde los estudiantes son llevados a un razonamiento con procesos que implican innovación, descubrimiento, desequilibrio con los saberes previos y construcción de nuevos conocimientos.

Esta intervención se hace ante la preocupación surgida por los resultados de las Pruebas Saber realizadas en la Institución Educativa Femenina Normal Manuel Cañizales, en el área de ciencias naturales, que de manera reiterativa en los últimos siete años desde el 2011 al 2018 muestra deficiencias en las competencias de uso comprensivo del conocimiento científico, explicación de fenómenos e indagación, por lo que mueve el mayor porcentaje de estudiantes con niveles de desempeño insuficiente y mínimo. En este sentido, se han evidenciado debilidades en procesos cognitivos, razón por la cual esta investigación se aborda desde tres momentos o fases: 1) Un diagnóstico o prueba de entrada que proporciona información sobre los conceptos e ideas previas que 
las estudiantes tienen sobre el método científico. 2) El diseño y aplicación de un ciclo de aprendizaje basado en el uso del método científico que permite la explicación de fenómenos empíricos vividos desde las realidades y contexto de las estudiantes. 3) Una evaluación o prueba de salida que permite comparar los resultados obtenidos antes y después de la intervención.

\section{Estado de conocimiento sobre el tema}

Aquí se describen las experiencias más representativas que sustentan la aplicación del método científico como estrategia pedagógica en esta investigación.

Rasilla (2004) en su trabajo del método científico como estrategia didáctica en la secundaria, afirma que en los colegios no hacemos ciencia, sino que la enseñamos a nuestros estudiantes, por lo cual se hace necesario que los alumnos, más que aprender ciencia, deben aprender a hacer ciencia. El método científico o experimental proporciona al alumno la oportunidad de saber cómo se hace el conocimiento.

Los pasos seguidos en los que se sustenta la estrategia pedagógica son: 1) Despertar el interés de los alumnos por el mundo de la ciencia. Esto conlleva a generar espacios de discusión con temas que despierten la curiosidad de los estudiantes. Para generar proximidad con el estudiante, se hace necesario conocer el trabajo real del hombre de ciencia y cómo se difumina el conocimiento. 2) Proporcionarles fascinación por el medio. 3) Después de conocer los medios de publicación, se explican detalladamente los pasos del método científico: definición del problema, obtención de información relacionada con el problema, formulación de hipótesis, diseño, realización e interpretación de experimentos y establecimiento de conclusiones. 4) Facilitar a los estudiantes posibles temas de investigación y. 5) La evaluación que permite proporcionar información al alumno, para mejorar y ampliar el conocimiento que tiene de sí mismo y de su trabajo.

Se concluye que el método científico como estrategia de aula:

1) Contribuye a mejorar la calidad en la enseñanza de las ciencias. 2) Facilita una renovación en las aulas de ciencias, promoviendo trabajos de investigación dirigida, donde los alumnos cuestionan su realidad mediante la emisión de hipótesis y diseño de experimentos. 3) El educador ha de promover la discusión y mediar en el proceso. 4) Esta forma de trabajo es enriquecedora, puesto que no genera en los estudiantes la idea de un conocimiento acabado y rígido, impuesto por parte del docente. 5) Este modo de trabajar en equipo en el que el educador es un mediador en todo el proceso permite una relación más cercana y dialogante, que estimula las relaciones interpersonales. 6) La motivación e interés de los alumnos por el proyecto los lleva a un mayor grado de exigencia. 7) El proceso de investigación es interdisciplinar, transversaliza todas las áreas fundamentales del plan curricular (Rasilla, 2004, p. 114). 


\section{Posteriormente Soler y Arteaga (2014) en su trabajo Aprendizaje global en el aula de cinco años basado en el método científico, plantean que:}

Enseñar ciencias resulta esencial para entender y conocer nuestra realidad, además ayuda a que los niños y niñas conecten con la importancia existente de la relación entre el progreso tecnológico y científico con la sociedad, concepto conocido como Enfoque CTS (Ciencia-Tecnología-Sociedad). También, se concibe la ciencia desde una perspectiva socioconstructivista. [...] Actualmente, desde el ámbito de la didáctica de las ciencias naturales, la enseñanza del método científico tiene sus detractores, se parte de la idea de que, al aplicar de manera rigurosa unas determinadas fases metodológicas (observación, formulación de hipótesis, experimentación...) para investigar un elemento o fenómeno, obtendremos como resultado el descubrimiento de la realidad. Desde esta concepción de método científico, se deja a un lado la importancia de la creatividad de los individuos que hacen ciencia, limitando con ello el conocimiento y transmitiendo la idea errónea de que se trata de una verdad absoluta, que como tal es incuestionable. La finalidad de esta propuesta de intervención en el aula, no está enfocada a desarrollar el método científico como una manera de descubrimiento de la verdad absoluta, sino como un instrumento válido para introducir a los niños y niñas en el mundo de las ciencias; ofreciéndoles una metodología de trabajo basada en la observación, búsqueda de información, ordenación de datos, formulación de preguntas para poder elaborar sus propias conclusiones, a través de la experimentación directa, facilitándoles el desarrollo de capacidades y competencias que puedan transferir a otras áreas, de manera que todas estas habilidades adquiridas contribuyan al desarrollo integral de los niños y niñas (pp. 670-673).

Para estudiar el comportamiento del caracol serrano se aplicó el método científico con el propósito de: 1) generar transversalidad en los contenidos que integran el currículo de Educación Infantil; 2) desarrollar habilidades científicas; y 3) generar una formación integral en los estudiantes.

Se concluye que, al realizar la intervención se logra verificar que la contribución optimiza el trabajo, el desarrollo de capacidades y competencias de los estudiantes. De esta manera, se fortalece el proceso de enseñanza - aprendizaje aportando la base para generar nuevo conocimiento.

Finalmente, Romero, Losada, Noriega y Martín (2017) afirman lo siguiente en su estrategia didáctica: para la enseñanza de la ciencia puntualizan lo siguiente:

La enseñanza de la ciencia requiere un itinerario a lo largo de las diferentes etapas educativas, que se inicie desde la manipulación y experimentación para posteriormente ir avanzando hasta llegar a la concreción y abstracción. La idea, sobre todo en las primeras edades, no es transmitir a los niños y niñas información o conocimientos científicos, sino proporcionarles experiencias significativas y sugerentes en relación con fenómenos o procesos del mundo físico y natural que les rodea y que les permitan entender aspectos presentes en su vida cotidiana. 
Más allá de la adquisición de contenidos, el trabajo en ciencia permite la adquisición de valores y destrezas, de competencias necesarias para el desarrollo integral del alumnado, como el trabajo cooperativo, la curiosidad, el sentido crítico, la rigurosidad en el trabajo, creatividad, imaginación [...] Pero el pensamiento científico no solo es positivo en el ámbito académico, sino que ofrece múltiples ventajas en la vida diaria y en la formación del niño como persona, va más allá y se relaciona también con la capacidad para ser autónomo o la resolución de problemas de la vida cotidiana. Una visión lógica desarrollada ayuda a los niños a encontrar relaciones entre los hechos, las ideas o las causas y los efectos.

Se precisa, entre otras cosas, con el desarrollo del pensamiento científico, el alumnado:

- Mejora su capacidad de razonamiento y su habilidad para pasar de nociones básicas a complejas

- Aprender a resolver problemas en situaciones reales

- Practica la construcción de su propio aprendizaje

- Ejercita su capacidad deductiva y aprende a crear estrategias y soluciones propias.

- Mejora su relación con el entorno físico y su percepción de los espacios, las formas, las partes y el todo.

Finalmente se puntualiza en que, para iniciar con los estudiantes a trabajar el método científico se tiene en cuenta:

- Aprender a realizar una revisión bibliográfica, enunciar hipótesis de trabajo que sean medibles y cuantificables, identificar y controlar variables, recoger datos y registrarlos, aprender a interpretar esos datos, extraer conclusiones objetivas y presentar resultados a través de: exposiciones orales, redacción de artículo científico, elaboración de póster resumen, elaboración de una presentación con TIC y divulgación del trabajo (pp. 8-10).

\section{Materiales y método}

La metodología utilizada en esta investigación es mixta. "Representa la integración sistemática de los métodos cuantitativo y cualitativo en un solo estudio, con el fin de obtener una fotografía más completa" (Sampieri, 2010, p. 546). El enfoque cuantitativo se presentó en la recolección de datos a través de las encuestas con sus respectiva tabulación y análisis de la información, las cuales fueron aplicadas en la etapa a priori y en la etapa a posteriori. También estuvo enmarcado en la orientación que muestra la investigación, partiendo de sus objetivos y la metodología de trabajo adelantada con las estudiantes en el aula de clase. En el enfoque cualitativo, Mejía (2004) "presenta una visión holística de la realidad" (p. 282). Además, "el plan de recolección de información es emergente y cambiante en función de los hallazgos realizados durante el avance del proceso investigativo" (Quintana, 2006, p. 64). Por lo cual, como se precisa en la tabla 1, este enfoque estuvo presente en los talleres de discusiones 
y el registro de observación en el aula. Dichos instrumentos permitieron una triangulación de la información, entendida como "el uso de múltiples métodos en el estudio de un mismo objeto" (Valencia, 2000, p. 15).

Partiendo de la permanente asistencia a clases en el grado Sexto B como criterio de selección, se trabajó con una población de 38 estudiantes y una muestra aleatoria de 20 , con edades entre 11 y 13 años.

Se utilizó un diseño exploratorio descriptivo que posibilitó que este estudio se convirtiera en referente o potencial fuente de información que amplíe posteriores investigaciones afines. En cuanto a su carácter descriptivo, se tuvo en cuenta la explicación de fenómenos empíricos para generar pensamiento crítico y reflexivo, planteado en esta investigación. Las técnicas utilizadas que ofrecieron la información requerida fueron la encuesta, la observación y los talleres y grupos de discusión.

Tabla 1. Técnicas e instrumentos de recolección de información

\begin{tabular}{|c|c|c|c|c|}
\hline Objetivos específicos & Actividades & $\begin{array}{l}\text { Técnicas de } \\
\text { recolección }\end{array}$ & $\begin{array}{c}\text { Instrumentos } \\
\text { de } \\
\text { recolección }\end{array}$ & Sistematización \\
\hline $\begin{array}{l}\text { Explorar mediante } \\
\text { prueba diagnóstica } \\
\text { los conocimientos } \\
\text { previos que las estu- } \\
\text { diantes tienen sobre } \\
\text { el método científico. }\end{array}$ & $\begin{array}{l}\text { Desarrollo de guía } \\
\text { diagnóstica. } \\
\text { Aplicación de en- } \\
\text { cuesta a estudiantes. }\end{array}$ & Encuesta. & $\begin{array}{l}\text { Cuestiona- } \\
\text { rio. }\end{array}$ & $\begin{array}{l}\text { Encuestas so- } \\
\text { bre conocimien- } \\
\text { tos previos de } \\
\text { las estudiantes. }\end{array}$ \\
\hline $\begin{array}{l}\text { Diseñar y aplicar un } \\
\text { ciclo de aprendizaje } \\
\text { basado en el uso del } \\
\text { método científico que } \\
\text { facilite el desarrollo } \\
\text { cognitivo en las es- } \\
\text { tudiantes. }\end{array}$ & $\begin{array}{l}\text { Lecturas de reflexión } \\
\text { y exploración, desa- } \\
\text { rrollo de talleres y ex- } \\
\text { posiciones. } \\
\text { Evaluación de se- } \\
\text { guimiento a ciclo de } \\
\text { aprendizaje. }\end{array}$ & $\begin{array}{l}\text { Observa- } \\
\text { ción. }\end{array}$ & $\begin{array}{l}\text { Lista de } \\
\text { cotejo. }\end{array}$ & $\begin{array}{l}\text { Fichas de se- } \\
\text { guimiento del } \\
\text { progreso de } \\
\text { las estudiantes } \\
\text { (Lista de cote- } \\
\text { jo). }\end{array}$ \\
\hline $\begin{array}{l}\text { Comparar los resulta- } \\
\text { dos a priori y a pos- } \\
\text { teriori que lleven a } \\
\text { verificar la construc- } \\
\text { ción de nuevos cono- } \\
\text { cimientos. }\end{array}$ & $\begin{array}{l}\text { Consultas, indaga- } \\
\text { ción, observación y } \\
\text { experimentación. } \\
\text { Aplicación y evalua- } \\
\text { ción: escoger un tema } \\
\text { o evento que despier- } \\
\text { te la curiosidad y ex- } \\
\text { plicarlo haciendo uso } \\
\text { del método científico. } \\
\text { Aplicación de en- } \\
\text { cuestas a posteriori. }\end{array}$ & $\begin{array}{l}\text { Talleres y } \\
\text { grupos de } \\
\text { discusión. } \\
\text { Encuesta. }\end{array}$ & $\begin{array}{l}\text { Registro de } \\
\text { observación } \\
\text { en el aula. } \\
\text { Cuestiona- } \\
\text { rio. }\end{array}$ & $\begin{array}{l}\text { Registro de ob- } \\
\text { servación en el } \\
\text { aula y encues- } \\
\text { tas realizadas } \\
\text { después de } \\
\text { aplicar el ciclo } \\
\text { de aprendizaje }\end{array}$ \\
\hline
\end{tabular}

Fuente: elaboración propia. 


\section{Resultados y discusión de resultados}

Se plantean los resultados obtenidos a partir de un análisis que se basa en los objetivos de esta investigación. A continuación, se describen los pasos que se siguieron:

1. Prueba de entrada

2. Aplicación del ciclo didáctico

3. Registro de observación en el aula

4. Prueba de salida

5. Comparativo entre la etapa a priori y a posteriori.

Para manejar la información cuantitativamente, se asignan unas categorías a las respuestas obtenidas en las encuestas y se establece relación entre estas categorías y los criterios de evaluación adoptados en la Institución Educativa que son: desempeño bajo (1 a 2,9), básico $(3,0$ a 3,9), alto $(4,0$ a 4,6$)$ y desempeño superior $(4,7$ a 5,0). Esta información se precisa en la figura 1.

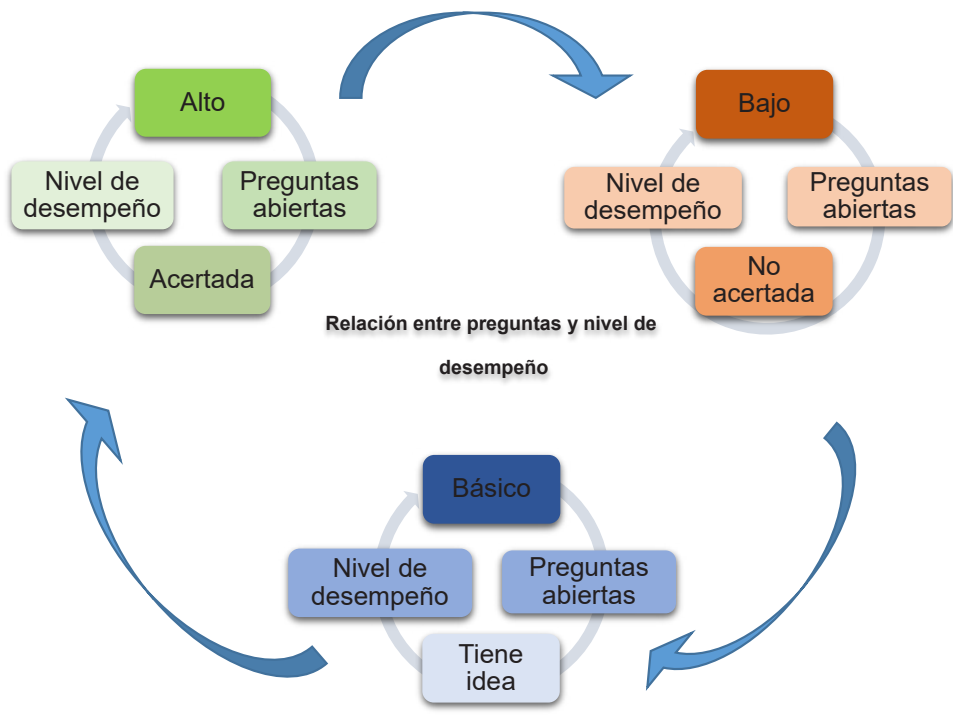

Figura 1. Relación entre preguntas y nivel de desempeño Fuente: elaboración propia.

\section{Prueba de entrada}

La primera medida para dar cuenta de la relevancia que tiene en todo proceso de enseñanza y aprendizaje es conocer las ideas previas de los estudiantes. 
En este caso, para tener un punto de referencia en el abordaje de contenidos referidos al método científico y saber las proximidades de las estudiantes con este método, se realizó una prueba de entrada o diagnóstica denominada etapa a priori (ver figura 2).

En esta etapa el $78 \%$ de las estudiantes no saben la respuesta de preguntas directamente relacionadas con el tema objeto de estudio. Lo anterior puede comprenderse, teniendo en cuenta que el tema es nuevo para ellas y las nociones que puedan presentarse no están estructuradas sobre conceptos sólidos adquiridos en el aula de clase.

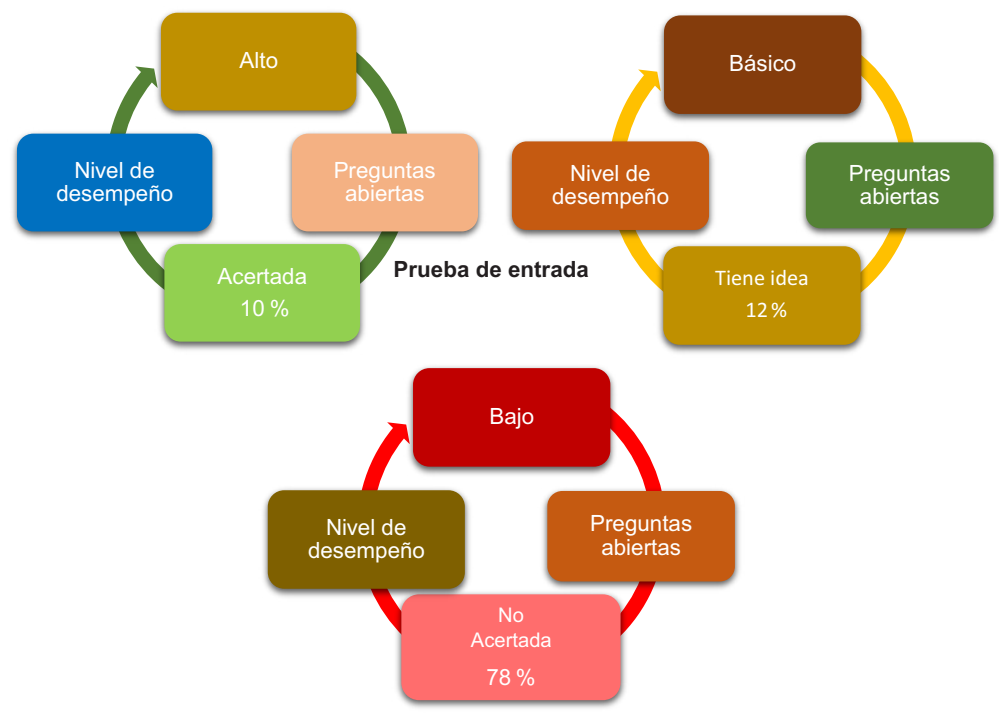

Figura 2. Nivel de desempeño relacionado con preguntas abiertas "prueba de entrada" Fuente: elaboración propia.

\section{Aplicación del ciclo didáctico "Ciencia y método científico"}

El ciclo didáctico se aplicó en dos fases. La primera comprendía los contenidos conceptuales abordados en 8 semanas. En la segunda fase las estudiantes debían escoger dos fenómenos empíricos (oxidación de las frutas y la maduración del aguacate en condiciones no climatéricas), y explicarlos haciendo uso de las etapas del método científico. Su tiempo de aplicación fue de 4 semanas.

En el fenómeno de la oxidación de las frutas se observó el comportamiento de la manzana, el banano, la sandía y la naranja bajo la influencia del aire.

Las estudiantes comprendieron que, al partir las frutas, las células presentes sufrían lesiones, quedando vulnerables ante ataques de agentes externos. 
Por esta razón las sustancias metabólicas o los fenoles eran expuestos, generando el cambio de color en las frutas (oxidación). Se dieron cuenta que ese efecto fenólico lo podían retrasar o evitar, añadiendo jugo de limón a las frutas expuestas en contacto con el aire.

En el segundo fenómeno, relacionado con el proceso de maduración del aguacate envuelto en papel periódico, debían tomar tres aguacates del mismo tamaño, envolver en papel periódico uno de los aguacates acompañado de un banano maduro. El segundo se debía envolver solo en el papel periódico, y el tercero se dejaba sin envolver. Haciendo uso de todos los órganos sensoriales, debían registrar los cambios que se presentaban, mientras maduraban los aguacates (Arbeláez, 2005).

Aquí concluyeron que el aguacate envuelto en papel periódico acompañado por el banano maduró más rápido que los otros debido a que algunas frutas maduras, en este caso el banano, contenía etileno y este pasaba al aguacate acelerando su maduración. En otras conclusiones agregaron que la tinta del periódico también contenía etileno y que este periódico emitía calor al aguacate y por eso la maduración se aceleraba.

Esta etapa estuvo acompañada de una lista de cotejo (ver tabla 2) y un registro de observación de trabajo en el aula necesarios para identificar cambios en las estudiantes en términos de logros y desempeños.

Tabla 2. Lista de cotejo basada en indicadores de desempeño

\section{Indicadores de desempeño}

Identifica conceptos básicos sobre ciencia

Reconoce y comprende la importancia del método científico en la explicación de fenómenos Identifica y aplica las etapas del método científico

\begin{tabular}{|c|c|c|c|c|c|c|}
\hline \multirow[t]{2}{*}{$\begin{array}{c}N^{\circ} \text { de } \\
\text { estudiantes }\end{array}$} & \multicolumn{2}{|c|}{$\begin{array}{l}\text { Comprende que todos } \\
\text { podemos hacer ciencia }\end{array}$} & \multicolumn{2}{|c|}{$\begin{array}{c}\text { Se apoya en el método científico } \\
\text { para explicar eventos o fenómenos } \\
\text { que llaman su atención }\end{array}$} & \multicolumn{2}{|c|}{$\begin{array}{l}\text { Participa en clase, } \\
\text { muestra dinamismo } \\
\text { y respeta las opiniones } \\
\text { de sus compañeras }\end{array}$} \\
\hline & SI & $\mathrm{NO}$ & SI & NO & SI & $\mathrm{NO}$ \\
\hline 1 & & $\mathrm{X}$ & & $\mathrm{X}$ & & $\mathrm{X}$ \\
\hline 2 & & $X$ & & $\mathrm{X}$ & & $\mathrm{X}$ \\
\hline 3 & $\mathrm{X}$ & & & & & \\
\hline 4 & & $X$ & & $\mathrm{X}$ & & $\mathrm{X}$ \\
\hline 5 & $X$ & & $\mathrm{X}$ & & $X$ & \\
\hline 6 & $X$ & & $X$ & & $X$ & \\
\hline 7 & $X$ & & $X$ & & $X$ & \\
\hline 8 & $X$ & & & $X$ & & $\mathrm{X}$ \\
\hline 9 & $X$ & & $\mathrm{X}$ & & $X$ & \\
\hline
\end{tabular}




\section{Indicadores de desempeño}

Identifica conceptos básicos sobre ciencia

Reconoce y comprende la importancia del método científico en la explicación de fenómenos Identifica y aplica las etapas del método científico

\begin{tabular}{|c|c|c|c|c|c|c|}
\hline \multirow[t]{2}{*}{$\begin{array}{l}N^{\circ} \text { de } \\
\text { estudiantes }\end{array}$} & \multicolumn{2}{|c|}{$\begin{array}{l}\text { Comprende que todos } \\
\text { podemos hacer ciencia }\end{array}$} & \multicolumn{2}{|c|}{$\begin{array}{c}\text { Se apoya en el método científico } \\
\text { para explicar eventos o fenómenos } \\
\text { que llaman su atención }\end{array}$} & \multicolumn{2}{|c|}{$\begin{array}{l}\text { Participa en clase, } \\
\text { muestra dinamismo } \\
\text { y respeta las opiniones } \\
\text { de sus compañeras }\end{array}$} \\
\hline & SI & NO & SI & NO & SI & NO \\
\hline 10 & $\mathrm{X}$ & & $\mathrm{X}$ & & $\mathrm{X}$ & \\
\hline 11 & $\mathrm{X}$ & & $\mathrm{X}$ & & $\mathrm{X}$ & \\
\hline 12 & $X$ & & $\mathrm{X}$ & & $X$ & \\
\hline 13 & $\mathrm{X}$ & & $\mathrm{X}$ & & $\mathrm{X}$ & \\
\hline 14 & $\mathrm{X}$ & & & $X$ & & $\mathrm{X}$ \\
\hline 15 & $X$ & & $\mathrm{X}$ & & $\mathrm{X}$ & \\
\hline 16 & $\mathrm{X}$ & & $\mathrm{X}$ & & $\mathrm{X}$ & \\
\hline 17 & $\mathrm{X}$ & & $\mathrm{X}$ & & $\mathrm{X}$ & \\
\hline 18 & & $X$ & & $\mathrm{X}$ & & $\mathrm{X}$ \\
\hline 19 & $\mathrm{X}$ & & $\mathrm{x}$ & & $\mathrm{X}$ & \\
\hline 20 & $x$ & & $\mathrm{X}$ & & $\mathrm{X}$ & \\
\hline 21 & $x$ & & $x$ & & $x$ & \\
\hline 22 & $\mathrm{X}$ & & $X$ & & $\mathrm{X}$ & \\
\hline 23 & $x$ & & $X$ & & $\mathrm{X}$ & \\
\hline 24 & $\mathrm{X}$ & & $\mathrm{X}$ & & $\mathrm{X}$ & \\
\hline 25 & $x$ & & $\mathrm{X}$ & & $\mathrm{X}$ & \\
\hline 26 & $X$ & & $\mathrm{X}$ & & $\mathrm{X}$ & \\
\hline 27 & $X$ & & $\mathrm{X}$ & & $X$ & \\
\hline 28 & $X$ & & $\mathrm{X}$ & & $X$ & \\
\hline 29 & $X$ & & $\mathrm{X}$ & & $X$ & \\
\hline 30 & $X$ & & $\mathrm{X}$ & & $\mathrm{X}$ & \\
\hline 31 & $X$ & & $\mathrm{X}$ & & $X$ & \\
\hline 32 & $x$ & & $\mathrm{X}$ & & $\mathrm{X}$ & \\
\hline 33 & $\mathrm{X}$ & & $\mathrm{X}$ & & $\mathrm{X}$ & \\
\hline 34 & $X$ & & $\mathrm{X}$ & & $\mathrm{X}$ & \\
\hline 35 & $X$ & & $\mathrm{X}$ & & $\mathrm{X}$ & \\
\hline 36 & $X$ & & $X$ & & $X$ & \\
\hline 37 & $X$ & & $\mathrm{X}$ & & $\mathrm{X}$ & \\
\hline 38 & $\mathrm{X}$ & & $\mathrm{X}$ & & $\mathrm{X}$ & \\
\hline
\end{tabular}

Fuente: elaboración propia. 


\section{Registro de observación en el aula}

En la figura 3 se describen los aspectos considerados positivos y negativos presentados durante la intervención, siendo estos últimos identificados como algunos limitantes en la investigación.

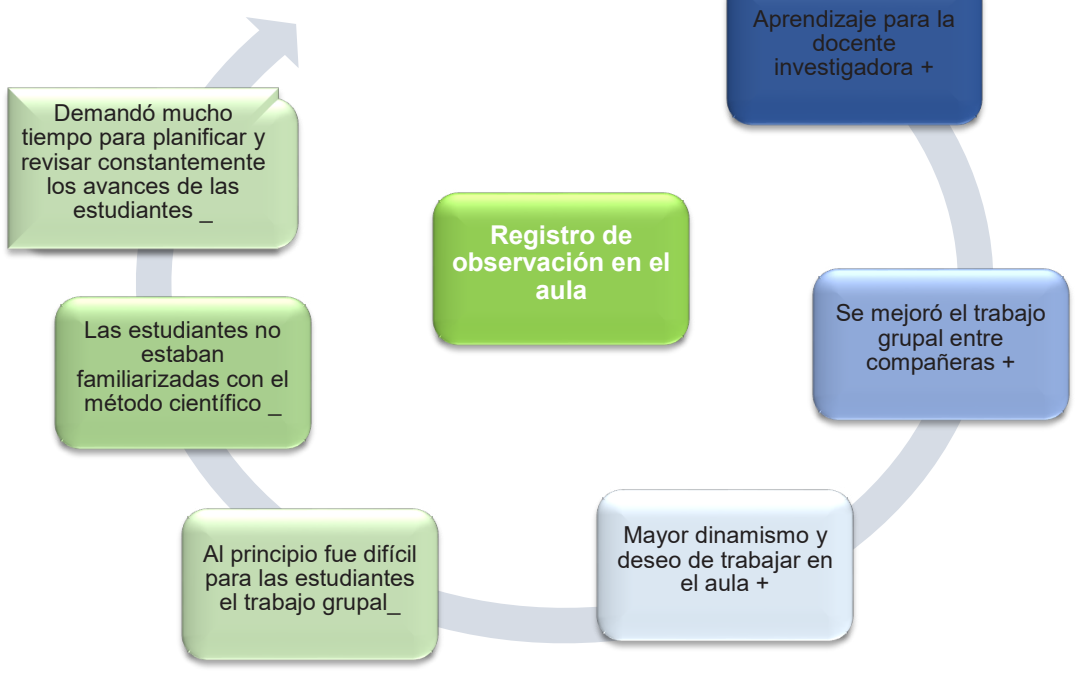

Figura 3. Registro de observación en el aula Fuente: adaptada de Gonzales (2012, p. 234).

\section{Prueba de salida}

Después de aplicar el ciclo didáctico, tuvo lugar una prueba de salida, denominada etapa a posteriori (ver figura 4). Se repitió el mismo procedimiento empleado en la etapa a priori o diagnóstica.

\section{Comparativo entre la etapa a priori y a posteriori}

En la tabla 3, se aprecia la relación establecida entre niveles e indicadores de desempeño, registrados en la lista de cotejo. Relación que se establece para identificar cambios representativos en términos de logros y competencias.

Tabla 3. Indicadores de desempeños tomados de lista de cotejo

\begin{tabular}{lll}
\hline \multicolumn{1}{c}{ Saber } & \multicolumn{1}{c}{ Saber hacer } & \multicolumn{1}{c}{ Saber ser } \\
\hline $\begin{array}{l}\text { Comprende que } \\
\text { todos podemos }\end{array}$ & Se apoya en el método científico & para explicar eventos o fenómenos \\
hacer ciencia & que llaman su atención & $\begin{array}{l}\text { mismo y respeta las opiniones de } \\
\text { sus compañeras }\end{array}$ \\
\hline
\end{tabular}



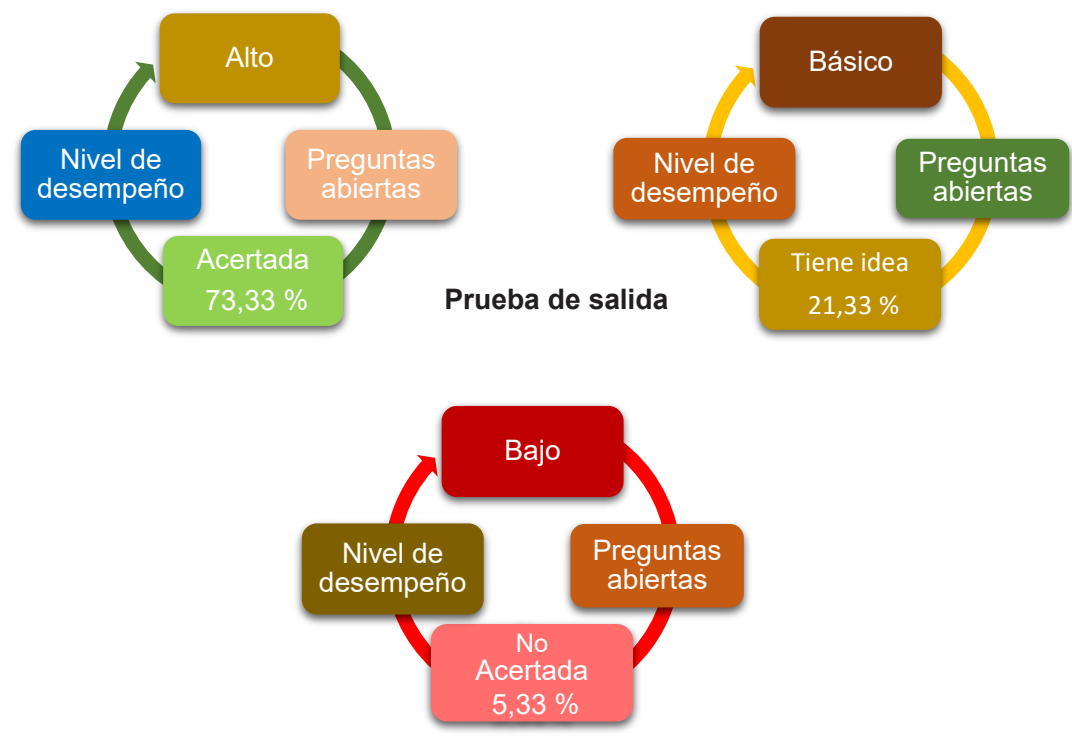

Figura 4. Nivel de desempeño relacionado con preguntas abiertas "prueba de salida" Fuente: elaboración propia.

Como se aprecia en la figura 5, al comparar los resultados obtenidos en la etapa de entrada con la etapa de salida, se puede ver como el nivel de desempeño alto, registrado en la etapa inicial, fue de $10 \%$ y pasó en la etapa de salida a $73,33 \%$. Este resultado lleva a entender que el 73,33 \% de las estudiantes logran comprender que todos podemos hacer ciencia, se apoyaron de manera apropiada en el método científico para explicar eventos o fenómenos que llamaron su atención y participaron en la clase mostrando dinamismo y respeto por las opiniones de sus compañeras.
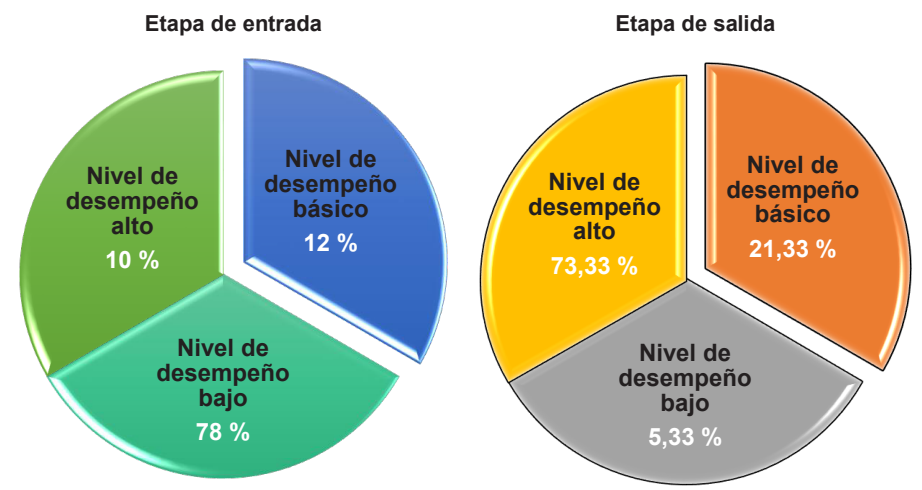

Figura 5. Comparativo etapa de entrada versus etapa de salida Fuente: elaboración propia. 
A continuación, se presenta en la tabla 4, un comparativo entre las etapas que plantea Mujica (2015) para alcanzar un desarrollo cognitivo en el aula y las actividades desarrolladas por las estudiantes durante la aplicación del ciclo didáctico en esta investigación:

Tabla 4. Etapas para alcanzar un desarrollo cognitivo en el aula

Actividades planteadas por Mujica (2015) para el desarrollo de procesos cognitivos en el aula
Actividades realizadas durante el ciclo didáctico en esta investigación que permitieron el desarrollo de procesos cognitivos en el aula (fase de aplicación)

Interacción y diálogo entre estudiantes. Trabajo grupal y cooperativo.

Aprendizaje ligado al pensamiento críti$\mathrm{co}$, hay que cuestionarse constantemente haciendo preguntas durante la clase.

Promover el pensamiento crítico y el aprendizaje activo y cooperativo: aprovechar lo que ya se sabe y autocorregirse Que hagan escuchas activa y participen en la clase y en las discusiones.

Trabajan en grupos pequeños, informan lo que han hecho y los problemas que han surgido.

Hay que transferir la responsabilidad de aprender del maestro a el estudiante.

Reflexionar frente a los contenidos para comprenderlos y aprenderlos.

Las estudiantes se plantean interrogantes frente al fenómeno de oxidación de las frutas y maduración del aguacate.

En torno a la explicación del fenómeno de oxidación de las frutas y maduración del aguacate, las estudiantes trabajan en grupo, sustentan sus avances de manera continua ante el resto de sus compañeras, surgen preguntas entre ellas y se da el espacio para correcciones y ajustes.

Durante el informe de los trabajos en grupo realizado por las estudiantes, estas hablan de las dificultades presentadas en la explicación del fenómeno de oxidación de las frutas.

La profesora investigadora fue una orientadora que permitió que las estudiantes llegaran por sí solas a sacar sus propias conclusiones.

Las estudiantes aplicaron las etapas del método científico para explicar los fenómenos planteados mostrando un avance significativo entre la etapa de entrada y la etapa de salida.

Activar los organizadores previos so- Se realizó una prueba diagnóstica sobre el mébre el tema. todo científico como tema.

Al final que los estudiantes expliquen qué han aprendido.

Se evidencia el aprendizaje mediante el trabajo que realizan las estudiantes explicando la oxidación de las frutas y maduración del aguacate. Finalmente, la confrontación de los resultados obtenidos en la prueba de entrada vs la prueba de salida.

Que los estudiantes opinen y evalúen el trabajo de sus compañeros.
Se pide a cada grupo la opinión frente al trabajo realizado por sus compañeras y se abre el espacio para controvertir y defender sus producciones. 
Además del desarrollo cognitivo alcanzado por las estudiantes, esta información da muestra de un aprendizaje significativo y duradero, la fijación de conceptos y la transversalidad del método científico cuando se trabaja en el aula. Muestra que las estudiantes se cuestionaron para definir un problema, plantearon estrategias y técnicas para darle solución, buscaron información relacionada con el problema, formularon hipótesis, realizaron predicciones en torno al problema y diseñaron sus experimentos, donde describieron procedimientos e interpretaron información para llegar a conclusiones. El trabajo fue dirigido y permitió que las estudiantes desarrollaran competencias argumentativas, propositivas y socializadoras.

De manera más precisa se puntualizan los cambios mostrados por las estudiantes al final de la intervención y que evidencian los resultados anteriores (ver tabla 5):

Tabla 5. Cambios reflejados en las estudiantes al final de la intervención

\section{Al principio de la intervención}

No lograban explicar e identificarse como metódicas o no metódicas.
Las que se consideraba metódicas argumentaron diciendo que eran ordenadas, que siempre hacían sus tareas, entregaban sus trabajos a tiempo, llevaban sus cuadernos al día, eran puntuales y que siempre terminaban las tareas asignadas cuando ayudaban en labores de su casa.

Por otro lado, las que no se consideraban metódicas argumentaban que eran desordenadas, que sus cuadernos no los llevaban al día y que no hacían ni entregaban tareas.

Mostraban dificultad para trabajar en equipo presentándose lo siguiente:

1. Manifestaban inconformidad porque alguna compañera no quería participar en los trabajos.

2. Por lazos de amistad incluían a compañeras que no trabajaban, lo cual se evidenciaba en las socializaciones de grupos.

En las competencias argumentativa y comunicativa se observaba mucha timidez y miedo para participar en clase.

Algunas niñas no mostraban mucho interés por la clase y eran sorprendidas haciendo actividades de otras asignaturas. se notaba más responsabilidad en el trabajo grupal, todas participaban en las realizaciones, lo cual era evidente cuando socializaban y respondían las preguntas que se les hacía.

Había participación constante de toda la clase, las niñas más tímidas y silenciosas empezaron a expresar sus ideas, les gustaba contar experiencias empíricas relacionadas con el tema de estudio. Todas querían ser escuchadas haciendo la clase muy dinámica.

Todas tenían su atención puesta en la clase porque estaban muy pendientes de lo que decían las compañeras para corregirla, controvertir su punto de vista o hacer un aporte. Hacían muchas preguntas y de las respuestas dadas surgían nuevas preguntas. 
Al principio de la intervención

No lograban relacionar el método científico con los eventos que acontecían en su contexto.

Al final de la intervención

Comprendieron que la ciencia no es exclusiva de los científicos y que ellas como estudiantes también podían hacer ciencia.

Lograron aplicar los pasos del método científico en la explicación de los dos fenómenos utilizados en esta investigación (oxidación de las frutas y maduración del aguacate en condiciones no climatéricas) y en otros fenómenos que identificaban dentro de su contexto, logrando de esta manera relacionar lo aprendido en el aula de clase con los eventos que acontecían en su diario vivir.

Fuente: elaboración propia.

Finalmente, con los resultados evidenciados en esta investigación se plantea: 1) Se estableció como punto de partida una ruta basada en la identificación de conocimientos previos, que luego facilitó el diseño y aplicación de un ciclo didáctico orientado a estructurar conocimientos referidos al método científico, sus etapas y sus aplicaciones en la explicación de fenómenos y situaciones ligadas a la cotidianidad y al accionar de las estudiantes, permitiéndoles la apropiación de un aprendizaje generado desde la construcción de sus propios diseños experimentales. 2) con esto se da cuenta del progreso que mostraron las estudiantes desde la etapa a priori o etapa inicial, caracterizada por un gran desconocimiento del tema, donde las estudiantes no contaban con recursos conceptuales suficientes ligados al desarrollo de unas competencias argumentativas e interpretativas. Sin embargo, estas herramientas fueron exploradas y puestas en evidencia durante la etapa de aplicación de este método las cuales permitieron que las estudiantes explicaran fenómenos muy conocidos por ellas y que de manera metódica describieran el comportamiento y cambios observados, poniendo en práctica las habilidades científicas de la abstracción, deducción, observación, análisis y síntesis. Se logro así la modelización de conceptos respecto a la aplicación del método científico, ya que antes de la aplicación las estudiantes mostraban confusión para identificar y explicar las etapas de este método.

\section{Conclusiones}

Con esta investigación se buscaba examinar la influencia del método científico para activar el pensamiento crítico y reflexivo en las estudiantes. Los resultados evidencian que se aplicaron los pasos de este método en la explicación de fenómenos reales y empíricos que dieron respuestas a interrogantes planteados como producto de la observación y experimentación. Se deduce que, con su aplicación en el aula, se abre paso a la discusión y el análisis, hay fortalecimiento del trabajo grupal y cooperativo, se retoman conocimientos y experiencias previas que luego son confrontadas con experiencias del momento, se organizan, 
estructuran y reelaboran nuevos conceptos de alta fijación. Esto hace que la metodología sea transversal y active en los estudiantes el pensamiento crítico y reflexivo, mejorando su capacidad para pensar y resolver problemas, que de acuerdo con las etapas planteadas por Mujica (2015), se conoce como desarrollo cognitivo.

El método científico como estrategia de trabajo en el aula posibilita un aprendizaje duradero y significativo. Su vinculación en la enseñanza no solo tiene aplicación en las ciencias naturales, sino en cualquier área del saber porque sirve para marcar distancia de ejercicios y problemas fácticos planteados en el aula de clase que se resuelven mecánicamente. El método científico sirve para mostrar que estos deben ser remplazados por problemas donde los estudiantes sean llevados a un razonamiento con procesos que impliquen innovación, descubrimiento, desequilibrio con los saberes previos, creatividad y, en definitiva, donde se marquen claramente los tres momentos del pensamiento científico: lo conocido, el conflicto entre lo conocido y lo nuevo y la restructuración o reorganización del nuevo conocimiento. De esta manera se llega a un conocimiento más avanzado que garantiza en el estudiante un pensamiento crítico que siempre lo lleve a la reflexión.

Como es planteado en los estándares básicos de competencias de ciencias naturales, se evidenció en la etapa de resultados que, al desarrollar en los estudiantes competencias científicas, es posible explorar hechos y fenómenos, analizar problemas, observar, recoger y organizar información relevante, utilizar diferentes métodos de análisis, evaluar los métodos y compartir resultados en el desarrollo de experiencias. Así se fortalecen procesos cognitivos los cuales se abrieron lugar en esta investigación a través de la aplicación del método científico como estrategia pedagógica.

\section{Agradecimientos}

Este artículo es resultado de investigación del proyecto Método Científico Como Estrategia Pedagógica Para Activar el Pensamiento Crítico y Reflexivo, el cual se realizó en el marco de la Maestría en Educación de la Universidad de Medellín, realizada en la ciudad de Quibdó (Chocó), bajo la dirección de Claudia Maria Maya Franco y tutoría de Hilderman Cardona-Rodas.

\section{Referencias}

Arbeláez. E. (2005). Viajeros ciencias 7. Bogotá, Colombia: Norma.

Díaz, F. (2003). Cognición situada y estrategias para el aprendizaje significativo. Revista Electrónica de Investigación Educativa, 5(2), 1-13. Recuperado de https://redie.uabc.mx/redie/article/ view/85/151 
Gonzales, C. (2012). Aplicación del ABP en estudios de grado de enfermería (Tesis doctoral). Universidad de Valladolid, España. Recuperado de https://uvadoc.uva.es/bitstream/handle/10324/2932/ TESIS332-130606.pdf;jsessionid=EF6AC9E5F38C660ED216CBE1CCE8254E?sequence=1

Mejía, J. (2004). Sobre la investigación cualitativa. Nuevos conceptos y campos de desarrollo. Revista Electrónica Researchgate, 8(13), 277-279. Recuperado de https://www.researchgate.net/ publication/268057878_Sobre_la_investigacion_cualitativa_Nuevos_conceptos_y_campos de_desarrollo

Mineducación (2004). Estándares Básicos de Competencias en Ciencias Naturales y Ciencias Sociales "Preguntar para aprender". Altablero, (30) Recuperado de https://www.mineducacion.gov. co/1621/propertyvalues-31329_tablero_pdf.pdf

Mujica, R. (2015). Los procesos cognitivos en el aula. Recuperado de https://www.iphe.gob.pa/ Ediciones-Anteriores-web/menu2/crelb/3-15/Los \%20Procesos.pdf

Quintana, A. (2006). Metodología de Investigación Científica Cualitativa. En Quintana, A. y Montgomery, W. (Eds.) Psicología: Tópicos de actualidad. (pp. 47-84). Lima, Perú: UNMSM.

Rasilla, F. (2004). El método científico como recurso pedagógico en el bachillerato: haciendo ciencia en clase de biología. Revista electrónica Pulso, (27), 112-115. Recuperado de https://ebuah.uah. es/dspace/handle/10017/5128

Romero, L., Noriega y M. (2017). Proyecto e-Nanociencia: propuesta didáctica para la enseñanza de la ciencia" basada en el método científico". (pp. 8-10). Andalucia, España.

Soler, E. y Arteaga, B. (2014). Aprendizaje global en el aula de cinco años basado en el método científico. Diálogo Educacional, 14(43), 670-673. Recuperado de https://www.researchgate.net/publication/269931938_Aprendizaje_global en_el aula_de_5 anos_basado_en_el_metodo_cientifico

Sampieri, R. (2010). Metodología de la investigación (5a ed.). Mexico: Mcgraw-Hill

Valencia, M. (2000). La triangulación metodológica: sus principios, alcances y limitaciones. Investigación y educación en enfermería, XVIII(1), 13-26 Recuperado de www.redalyc.org/articulo. oa?id=105218294001 